\title{
Single-incision laparoscopic cholecystectomy: is it more than a challenge?
}

\author{
Antonio Iannelli • Anne Sophie Schneck • \\ Jean Gugenheim
}

Published online: 8 July 2010

(C) Springer Science+Business Media, LLC 2010

To the Editor,

We read with interest the paper by Ersin et al. [1] on single-incision laparoscopic cholecystectomy (SILS) cholecystectomy, which further proves the feasibility of this new surgical technique. However, we believe that some points need further discussion.

The first point concerns the issue of gallbladder suspension with transfixing suture. The authors state that for favorable results the suspending suture must be placed in the thoracoabdominal region, i.e., through an intercostal space. Despite obeying the rules of chest tube insertion, passing the suture in the pleural space may give rise to bleeding, pneumothorax, and/or lung injury, especially in a patient who is ventilated under positive pressure. The suspending suture, thus, should be better placed through the abdominal wall as suggested by Tacchino et al. [2]. The potential complications that may follow are not acceptable for a standard laparoscopic cholecystectomy.

Second, the possibility of making a cholangiography was not considered by the authors. Although the place of the cholangiography during laparoscopic cholecystectomy is still debated, evidence of the technical feasibility of the cholangiography during SILS cholecystectomy must be provided. This is of mainstay importance for the acceptance of the SILS cholecystectomy as a new technique. In fact, we believe that the decision not to perform intraoperative cholangiography must be made by the surgeon and not dictated by the limitations of the technique [3].

Third, the duration of surgery does not seem to be significantly different among the 20 procedures reported by the authors. Furthermore, reported operative times are by far longer than those currently needed for standard laparoscopic cholecystectomy in the absence of known intraoperative difficulties, such as cholecystitis, which were not recorded by the authors.

Disclosures Drs. Antonio Iannelli, Anne Sophie Schneck, and Jean Gugenheim have no conflicts of interest or financial ties to disclose.

\section{References}

1. Ersin S, Ozgur Firat O, Sozbilen M (2009) Single-incision laparoscopic cholecystectomy: is it more than a challenge? Surg Endosc 24:68-71

2. Tacchino R, Greco F, Matera D (2008) Single-incision laparoscopic cholecystectomy: surgery without a visible scar. Surg Endosc 23:896-899

3. Curcillo PG, Wu AS, Podolsky ER et al (2010) Single port access $\left(\mathrm{SPA}^{\mathrm{TM}}\right)$ cholecystectomy: a multi-institutional report of the first 297 cases. Surg Endosc. doi:10.1007/s00464-009-0856-x. [Epub ahead of print]
A. Iannelli $(\bowtie) \cdot$ A. S. Schneck · J. Gugenheim

Service de Chirurgie Digestive et Transplantation Hépatique,

Hôpital Archet 2, CHU de Nice, 151 route Saint Antoine de

Ginestiere, BP 3079, 06202 Nice, France

e-mail: antonio_iannelli@hotmail.com

A. Iannelli · A. S. Schneck · J. Gugenheim

Faculté de Médecine, Université de Nice-Sophia-Antipolis, 06107 Nice, France 